\title{
Microfluidics for nano-pathophysiology
}

\author{
Kae Sato ${ }^{1, *}$, Naoki Sasaki ${ }^{2}$, Helene Andersson $\mathrm{Svahn}^{3}$, Kiichi Sato ${ }^{4}$ \\ ${ }^{1}$ Department of Chemical and Biological Sciences, Faculty of Science, Japan \\ Women's University, Bunkyo, Tokyo 112-8681, Japan \\ ${ }^{2}$ Faculty of Science and Engineering, Toyo University, Kawagoe, Saitama 350-8585, \\ Japan \\ ${ }^{3}$ School of Biotechnology, Royal Institute of Technology, AlbaNova University Center, \\ 10691 Stockholm, Sweden \\ ${ }^{4}$ Division of Molecular Science, Faculty of Science and Technology, Gunma \\ University, Kiryu, Gunma 376-8515, Japan
}

* Corresponding author: fax +81-3-5981-3661, E-mail: satouk@fc.jwu.ac.jp

\begin{abstract}
Nanotechnology-based drug delivery systems hold promise for innovative medical treatment of cancers. While drug materials are constantly under development, there are no practical cell-based models to assess whether these materials can reach the target tissue. Recently developed microfluidics systems have revolutionized cell-based experiments. In these systems, vascular endothelial cells and interstitium are set in microchannels that mimic microvessels. Drug permeability can be assayed in these blood vessel models under fluidic conditions that mimic blood flow. In this review, we describe device fabrication, disease model development, nanoparticle permeability assays, and the potential utility of these systems in the future.
\end{abstract}




\section{Introduction}

Nano drug delivery systems (nanoDDS) were developed to maximize drug efficacy by ensuring precise dosing and targeting [1-3]. In the microcirculation systems of tumors, vascular permeability increases and the lymphatic system is underdeveloped. Under these conditions, nanodrugs accumulate and act on the target. Recently, however, nanodrug-insensitive foci have been reported [4]. In order to develop novel nanodrugs for these resistant foci, it is important to understand their microvascular physiology.

Two approaches have been used to evaluate nanodrugs: whole-body studies using test animals and detailed analyses of single cultured cell lines. In the animal experiments, cell-level analyses for histochemical and cardiovascular physiological studies are difficult. On the other hand, it is impossible for monolayer cultures of vascular endothelial cells to mimic biological reactions in the human body, with its many tissues and complex blood flow. Therefore, novel cell culture-based analytical methods are required to explain the physiological findings of animal experiments. Current monolayer cell culture systems lack a mechanism to generate fluidic shear stress mimicking blood flow and microenvironments such as interstitial tissues and intercellular effects.

Recent efforts to develop human-mimicking models with microfluidic devices have attracted attention [5-12]. In these devices, cells can be cultured in tube-shaped microchannels under shear stress conditions created by a fluidic pump. Use of transparent materials enables real-time observation and analyses. Micromodels mimicking organs and tissues such as liver [13], lung [14-17], gut [18-21], and vascular vessels [9] have been reported. These mimics may have practical utility in nanodrugs 
research.

This review discusses recent trends in disease models with a focus on blood vessel models in microfluidic devices. The basic elements of microfluidic disease models including device fabrication and choice of cell line are summarized; device-based histochemical and physiological analyses are also described in the context of their potential utility in nanodrug development.

2. Target pathophysiological processes in blood vessels

Models of sickle cell disease, infectious diseases, and vascular disorders associated with diabetes and cancer must be reconstituted from biological reactions that occur in complex tissues with blood flow, which cannot be mimicked by monolayer cultures of vascular endothelial cells. In sickle cell disease, erythrocytes have an unusual morphology and show low deformability, resulting in microcirculatory failure. Wood et al. proposed an assessment of the severity of sickle cell disease with a device mimicking erythrocyte flow [22]. Apoptosis of vascular endothelial cells has been monitored in a diabetes-mimicking device [23].

Changes in endothelial barrier function are strongly associated with inflammation caused by a variety of diseases and tumor cell invasion of blood vessels. Endothelial permeability tests are necessary to analyze these diseases and drug permeability. The vascular walls of solid cancers and inflamed sites have gaps tens of nanometers wide between endothelial cells [24]. These vascular walls were thought to be more permeable than normal walls because of these gaps. Models of blood vessels in tumor tissues 
cannot be achieved in monolayer culture systems and are desirable in DDS research.

To achieve these pathophysiological properties, microfluidic vascular models should have following characteristics: three-dimensional tubing, consisting of vascular and lymphatic endothelial cells, interstitial tissue, liquid flow mimicking blood flow, chemical stimulation by inflammatory cytokines, microscopic imaging, and real-time monitoring of permeation from the vessels. Structures of typical vascular models are shown in the following chapter.

3. Structure of the device

\subsection{Cells}

Because wide varieties of normal human vascular endothelial cells are commercially available, it is not difficult to develop vascular models. Human umbilical vein endothelial cells (HUVEC) are most frequently used in microfluidic models [25-31]. Human aortic endothelial cells (HAEC) were chosen for an atherosclerosis model [32, 33] and human pulmonary microvascular endothelial cells were cultured for a lung model [16]. In some microfluidic models, human dermal microvascular endothelial cells (HMVEC-d or HDMEC) have been used to mimic a microvascular system [26,34-37]. Non-human cells such as bovine aortic endothelial cells [38], bovine pulmonary artery endothelial cells (bPAEC) [39], or primary porcine aortic endothelial cells (PAEC) [40] were used because of their manageability. Some immortalized cell lines including human microvascular endothelial cell line HMEC-1 [41-44], human vascular 
endothelial cell line CRL1730 [45], mouse brain-derived endothelial cell line bEnd.3 [46], and Rat Brain Endothelial cell line RBE4 [47] have also been used.

\subsection{Materials}

Most microfluidic devices for vascular models are made of a silicone elastomer, poly(dimethylsiloxane) (PDMS). PDMS devices are produced by a soft lithography replica molding method, in which the devices are replicated from a reusable mold. The fabrication process is shown in Fig. 1. The mold is usually made of silicon or glass wafers with micrometer-size structures produced by photolithography with the epoxybased, photo-crosslinkable polymer SU-8 (Fig. 1a-c). A PDMS prepolymer is poured on top of this mold, allowed to solidify by heating (Fig. 1d), and then peeled from the mold (Fig. 1e). In order to create sealed microchannels, the PDMS replica is bound to another PDMS sheet or glass slide (Fig. 1f). Access holes are made in the closed microchannel and tubes are connected to pump fluid into the channels. While the mold master in this method is reusable, the microfluidic devices are discarded after every experiment because the materials are cheap.

Microfluidic devices for vascular models could be fabricated by laminating the PDMS sheets with microchannels and a porous membrane, used in cell culture inserts or Transwells (Fig. 2a). In these devices, 2 adjacent microchannels are separated by the membrane. Vascular endothelial cells were cultured on the membrane under fluidic conditions and vascular permeability could be assayed.

On the other hand, three-dimensional models, which more closely represent the human 
body, have also been reported. Three-dimensional cell culture in the microchannels was achieved by growing cells at the edge of a hydrogel matrix, allowing them to migrate into the gel. The device consisted of a cell culture channel filled with a hydrogel matrix, and a cell introduction channel (Fig. 2b). These channels were connected by small windows. Endothelial cells attached to the surface of the hydrogen matrix at the windows, and then migrated into the hydrogel to form a three-dimensional blood vessel model. Matrigel [48], type-1 collagen [49-51], collagen with fibronectin [52], or fibrin gel [31] have been used as hydrogel matrices. Hydrogel-based microchannels have been developed as a blood vessel model [26, 34].

Two-dimensional culture is more convenient, because cells can easily be supplied with fresh culture medium. Moreover, it is more compatible with microscopic imaging. Microfluidic devices enable replenishment of medium and microscopic imaging is relatively easy to achieve even in a three-dimensional culture environment, because the microfluidic devices are designed for fluidic operations and are small enough to be observed with a microscope.

\subsection{Pumping methods}

Pumping of fluids into a microchannel is important to construct pathological models in microfluidic devices. For example, it is necessary to pump fresh medium for perfusion culture of vascular cells in the microchannel, which acts as an artificial blood vessel. Pumping is also important to mimic blood flow and to introduce reagents.

A number of pumping methods have been reported. Pumping by hydrostatic pressure, 
which is generated by a difference in height between the inlet and outlet, can induce continuous flow with a simple experimental setup [26, 34, 53]. A computer-controlled pump can induce continuous flow and pulsatile flow at various rates. Syringe and peristaltic pumps have been utilized. Such pumps have been used to expose endothelial cells to continuous shear stress [30], pulsatile shear stress [25, 45], or both [54].

Although these pumping methods have been applied to cell experiments in microfluidic devices, commercially available pumps are generally much larger than the devices, and downsizing of the whole system is often limited by the size of the pumps. A solution to this limitation is to use small pumps. An integrated culture system driven by a small peristaltic pump, medium reservoir, and motor controller has been reported [55]. All of the components except the motor controller are integrated on a microscope stage insert, and the system combined with a microscope has been employed for field experimentation. A palmtop-sized microfluidic cell culture system driven by a miniaturized peristaltic pump has also been reported [44]. The pump possesses a reservoir for culture medium, an electrical control circuit, and an internal battery, so there is no need for tubes or electrical connections outside the system. Another solution to the limitation of downsizing is to integrate pumping units on microfluidic devices. Microfabricated peristaltic pumps have been utilized to analyze secreted molecules [56], to culture and analyze cells $[20,29]$, and to culture cells in microchambers in an automated manner [57]. A micropump driven by the mechanical pins of Braille display, which is an electro-mechanical device for displaying Braille characters by means of round-tipped pins for the visually impaired people, has been utilized for endothelial cell culture [36] and the development of a palm-sized cell culture system [58]. 


\section{Analyses with disease models}

\subsection{Mimicking blood flow}

There are many reports on the effects of shear stress, angiogenesis, and endothelial barrier function in microfluidic vascular disease models. Cellular experiments using these models under various shear stresses have been reported.

For example, shear stress levels from 0.7 to $130 \mathrm{dyn} / \mathrm{cm}^{2}$ were simultaneously applied in a single chip containing 10 microscale flow chambers, each $200 \mu \mathrm{m}$ wide and 100 $\mu \mathrm{m}$ deep [25]. A microscale parallel-plate flow chamber that generates a linear gradient of shear stress has also been reported [59]. This study showed that tumor necrosis factor alpha (TNF- $\alpha$ )-induced VCAM-1 and E-selectin expression was upregulated at low shear stress and suppressed at high shear stress.

Blood vessels perform their functions by vasodilation or vasoconstriction. By using elastic materials for microfluidic vascular models, pressure-regulated vasodilation can mimic the biological environment [33]. In this system, heart attack, hypertension, and heart rate changes are mimicked by means of a peristaltic pump. The micromodel simulates 6 conditions including normal, heart failure, hypertension, hypotension, tachycardia/exercise, and bradycardia, to generate physiologically relevant conditions. For each condition, pressure, flow, strain, and shear stress waveforms were closely matched to clinically observed values. Cells in the endothelial model showed increased F-actin, alignment in the direction of flow, and higher and continuous expression of cell adhesion molecule -catenin in comparison to static controls. 


\subsection{Angiogenesis}

Endothelial cell migration is features of angiogenesis and cancer metastasis. While only two-dimensional culture can be achieved with conventional transwell methods, cells could be cultured in three-dimensional structures in the microfluidic models. Biomimetic models could be constructed by mimicking blood flow and generating chemical concentration gradients.

For example, 3 microchannels, 1 for endothelial cell culture, and 2 filled with hydrogel and culture medium containing VEGF, were aligned and connected via small access windows $[50,51]$ (Fig. 3a). When the VEGF concentration gradient was generated in the hydrogel channel, the cells migrated and invaded the hydrogel toward the highVEGF side.

These devices were used to investigate the induction of blood vessels in tumors. Migration of endothelial cells toward the tumor cell side was observed by means of a micromodel in which endothelial, hydrogel, and tumor channels were aligned [49].

Song et al. reported the relationship between angiogenesis and shear stress [52]. Whereas HUVEC cultured in a micromodel migrated to the high-VEGF side and invaded the gel, invasion was not observed when the cells were subjected to strong shear stress. Moreover, inhibition of cell migration was regulated with NO.

\subsection{Barrier function}


The barrier function of vascular endothelial cells differs in static and flow culture. Shao et al. reported that albumin permeability increased under pulsed flow conditions due to static diffusion and hydraulic permeability [29].

It has also been reported that permeability decreases with increasing flow rate [37]. When endothelial cells were cultured in a microfluidic channel made of collagen gel under different fluidic conditions, the permeability coefficient of BSA or dextran decreased at high flow rates. Endothelial cells grown under higher flow rates displayed longer segments of VE-cadherin, suggesting flow might improve barrier function.

\subsection{Cancer metastasis}

Tumor metastasis is triggered by penetration of tumor cells into blood vessels. Circulating tumor cells migrate outside blood vessels to invade tissue. Microfluidic models can mimic this process (Fig. 3b). Zervantonakis et al. studied invasion of carcinoma and fibrosarcoma by means of three-dimensional vascular models with hydrogel [60]. The results suggested invasion of tumor cells was facilitated when the barrier function of the endothelial cells was weakened by TNF- from macrophages. Zang et al. demonstrated that adenoid cystic carcinoma cell aggregates transmigrated across the endothelium under the stimulation of chemokine CXCL 12[61]. These devices are applicable for analysis of the mechanisms underlying metastasis in addition to potential application for testing of anti-metastasis agents. 


\subsection{Evaluation of nanoparticle permeation}

Nanoparticles $>40 \mathrm{kDa}$, or with a diameter of around $100 \mathrm{~nm}$, are employed for tumorselective drug delivery, because they enhance permeability and retention [3]. Tumor blood vessels are highly permeable to the nanoparticles, mainly because of the porous structure of the vascular walls as shown by scanning electron microscopy [24]. However, permeability is governed by various parameters, including pore size and density and nanoparticle size. In addition, permeability has been determined by animal testing, which is less desirable due to cost, time, and ethical considerations. Transwell or cell culture inserts have been utilized to study the permeability of endothelial monolayers, the most important component of blood vessels. However, this technique has several disadvantages: (i) the size of the Transwell is far from that of tumor blood vessels; (ii) shear stress generated by blood flow cannot be applied to the monolayers; and (iii) a large amount of medium and reagents is consumed.

Microfluidic devices have been utilized as an experimental model to evaluate nanoparticle permeation through vascular walls (Fig. 3c). Microfluidic devices integrated with porous membranes have been used to culture endothelial cells on the membranes and evaluate permeability to fluorescence-labeled albumin ( 66 kDa) [40]. The permeability of the membrane without cells was consistent with a theoretical value. The permeability of the membrane covered with endothelial cells was also obtained and discussed with reported values.

The microfluidic devices have layered structures to sandwich the membranes between device substrates. To simplify the fabrication process and facilitate microscopic observation, planar microfluidic devices have also been developed. In these devices, 
cells were positioned with the aid of channel structures. For example, narrow microchannels $(3 \mu \mathrm{m} \times 3 \mu \mathrm{m})$ have been employed to connect a microchannel and microchamber, and to retain endothelial cells only in the microchannel [47]. Permeation of fluorescence-labeled dextran $(3 \sim 5 \mathrm{kDa})$ from the microchannel to the microchamber was studied, and the use of astrocyte-conditioned medium reduced the permeability of the endothelium. This result suggests the microdevice can test interactions between the endothelium and secreted materials from astrocytes in the medium, and the device can serve as a model of Blood-brain barrier (BBB).

Microposts several hundred micrometers long were used to confine a mixture of fibroblasts and Matrigel to a section of the microfluidic channels [48]. The mixture was considered a model of the interstitium, and permeation of fluorescence-labeled nanoparticles through the mixture was studied under static and flow conditions. The nanoparticles permeated in a size-dependent manner, and flow enhanced permeation.

Blood vessels have bifurcations and curvatures, which are mimicked by microfluidic models used to investigate particle-cell interactions. These studies suggest the complex and heterogeneous flow patterns encountered by particles in microvascular networks show a significantly higher propensity to adhere near bifurcations in comparison to branches of the microvascular networks.

Microfluidics must be useful for rapid evaluation of nanoparticles in vitro under microenvironments that mimic in vivo conditions. Furthermore, some systems could be used to evaluate in vivo toxicity of nanoparticles or for drug screening.

A microfluidic model was developed to assess thrombolytic agents that are selectively activated at obstructed vessels (Fig. 3d) [62]. In this model, microscale aggregates of nanoparticles were fabricated to break up into nanoparticles when exposed to 
abnormally high shear stress. The microfluidic stenosis model of PDMS has a constriction channel. In the model, a drug in the nanoparticle aggregates did not exhibit activity before the stenosis, but became active after breakup by the constricted channel.

\section{Complex micromodels}

\subsection{Blood-brain barrier models}

Blood Brain Barrier (BBB) is an interface that separates the brain from the circulatory system to protect the brain from toxic chemicals while regulating transport of essential molecules. Investigations about characteristics of several kinds of cells forming BBB are important to reveal the drug delivery to the brain.

Recently, some microfluidic BBB models were reported. In addition to micromodels with human brain (hCMEC/D3) [63] or rat brain endothelial cell line (RBE4) [47], complex microsystems have been developed in which mouse endothelial (b.END3) and astrocytic cell lines (C8-D1A) were co-cultured (Fig. 4a) [64].

In the complex system, key properties were tested by optical imaging, trans-endothelial electrical resistance (TEER), and permeability assays. The system mimicked the dynamic cerebrovascular environment with shear stress, and the results suggest the model expresses key characteristics of BBB.

The micromodel showed significantly higher TEER levels than static models. Permeability assays with a wide range of tracer sizes to demonstrate selective permeability indicated that the system is useful for studies of BBB function and delivery. It can be used to monitor changes in barrier function in response to various 
environmental stimuli.

Development of complex micromodels that reproduce organ-level pathophysiological responses will be beneficial to many fields that rely on animal testing, such as toxicology and drug discovery. Because these microsystems can be easily multiplexed and automated, they might be useful for high-throughput analysis and screening of cellular responses to nanoDDS.

\subsection{Lung models}

Simple microfluidic vascular models are not suitable for complex ex vivo models that mimic the nature of the tissue and differ from practical medicobiological requirements, because practical diseases are complicated by numerous factors. Recently, complex microdevices with 2 kinds of cells were proposed.

Development of a lung model is important for studies of infectious disease. Microfluidic lung models with alveolar epithelial cells $[14,15]$ and more complicated devices in which capillary endothelial cells and alveolar epithelial cells were cocultured have been proposed (Fig. 4b) [16, 17].

These devices contain 2 closely apposed microchannels separated by a thin $(10 \mu \mathrm{m})$, porous, flexible membrane of PDMS. The intervening membrane is coated with fibronectin or collagen; human alveolar epithelial cells and human pulmonary microvascular endothelial cells were cultured on opposite sides of the membrane.

The microsystem reconstituted the critical functional alveolar capillary interface of the human lung and reproduces organ-level responses to bacteria and inflammatory 
cytokines introduced into the alveolar space.

In nanotoxicology studies, the microdevice revealed that cyclic mechanical strain accentuates toxic and inflammatory responses to silica nanoparticles. Mechanical strain also enhances epithelial and endothelial uptake of nanoparticulates and stimulates transport into the underlying microvascular channel.

\section{Conclusions}

Blood vessel and organ models can be developed by a combination of a microfluidic device and cultured cells with extracellular matrices. Inherent cellular responses can be observed in microenvironments that mimic the human body with chemical stimulation under fluidic conditions that simulate blood flow. In order to demonstrate their utility as an assay device for nanodrugs, model functions must be compared to the human body. Moreover, detailed biochemical and molecular biological experiments should be performed with these micromodels. Because the amount of cells cultured in the microdevices is small, analyses of mRNA and proteins by RT-PCR or western blotting are difficult. However, these analyses will be feasible in the near future by rapid development of the analytical methods in microfluidic devices.

\section{Acknowledgment}

This work was supported in part by a Grant-in-Aid for Scientific Research (KAKENHI; Grant Number 24350035) and JST CREST (Core Research for Evolutional Science \& 
Technology). 
Figure captions

Figure 1. Fabrication process of PDMS microfluidic devices.

a) Photoresist, SU8 is spin coated onto a silicon wafer, b) UV light is exposed through a photomask, c) non cross-linked photoresist is removed, d) a PDMS prepolymer mixture is casted on the master, e) the hardened PDMS sheet is pulled off the mold, f) the PDMS sheet is bound to a flat substrate to form microchannels.

Figure 2. Microfluidic devices for 3D culture.

a) PDMS microfluidic device with a porous membrane. Two microchannels is separated by a thin porous membrane, b) PDMS microfluidic device with a hydrogel.

Figure 3. Illustration of disease models.

a) Microfluidic angiogenic sprouting model. Endothelial cells migrated into a gel, b) microfluidic tumor-vascular interface models for tumor cell intravasation and extravasation studies, c) nanoparticle permeation model, d) in vitro modeling of the microvascular occlusion and thrombosis model.

Figure 4. Illustration of complex micromodels.

a) Blood-brain barrier model reprinted from [64] with permission, b) Lung model reprinted from [16] with permission. 
References

[1] Y. Matsumura, H. Maeda, A new concept for macromolecular therapeutics in cancer chemotherapy: mechanism of tumoritropic accumulation of proteins and the antitumor agent smancs, Cancer Res, 46 (1986) 6387-6392.

[2] H. Maeda, Y. Matsumura, Tumoritropic and lymphotropic principles of macromolecular drugs, Crit Rev Ther Drug Carrier Syst, 6 (1989) 193-210.

[3] H. Maeda, H. Nakamura, J. Fang, The EPR effect for macromolecular drug delivery to solid tumors: Improvement of tumor uptake, lowering of systemic toxicity, and distinct tumor imaging in vivo, Adv Drug Deliv Rev, 65 (2013) 71-79.

[4] L. Zhang, H. Nishihara, M.R. Kano, Pericyte-coverage of human tumor vasculature and nanoparticle permeability, Biol Pharm Bull, 35 (2012) 761-766.

[5] M.H. Wu, S.B. Huang, G.B. Lee, Microfluidic cell culture systems for drug research, Lab Chip, 10 (2010) 939-956.

[6] D. Huh, G.A. Hamilton, D.E. Ingber, From 3D cell culture to organs-on-chips, Trends Cell Biol, 21 (2011) 745-754.

[7] N.K. Inamdar, J.T. Borenstein, Microfluidic cell culture models for tissue engineering, Curr Opin Biotechnol, 22 (2011) 681-689.

[8] M.B. Esch, T.L. King, M.L. Shuler, The role of body-on-a-chip devices in drug and toxicity studies, Annu Rev Biomed Eng, 13 (2011) 55-72.

[9] K.H. Wong, J.M. Chan, R.D. Kamm, J. Tien, Microfluidic models of vascular functions, Annu Rev Biomed Eng, 14 (2012) 205-230.

[10] D. Huh, Y.S. Torisawa, G.A. Hamilton, H.J. Kim, D.E. Ingber, Microengineered physiological biomimicry: organs-on-chips, Lab Chip, 12 (2012) 2156-2164. 
[11] A.D. van der Meer, A. van den Berg, Organs-on-chips: breaking the in vitro impasse, Integr Biol (Camb), 4 (2012) 461-470.

[12] J.H. Sung, M.B. Esch, J.M. Prot, C.J. Long, A. Smith, J.J. Hickman, M.L. Shuler, Microfabricated mammalian organ systems and their integration into models of whole animals and humans, Lab Chip, 13 (2013) 1201-1212.

[13] P.M. van Midwoud, E. Verpoorte, G.M. Groothuis, Microfluidic devices for in vitro studies on liver drug metabolism and toxicity, Integr Biol (Camb), 3 (2011) 509-521.

[14] D. Huh, H. Fujioka, Y.C. Tung, N. Futai, R. Paine, 3rd, J.B. Grotberg, S. Takayama, Acoustically detectable cellular-level lung injury induced by fluid mechanical stresses in microfluidic airway systems, Proc Natl Acad Sci U S A, 104 (2007) 18886-18891.

[15] D.D. Nalayanda, C. Puleo, W.B. Fulton, L.M. Sharpe, T.H. Wang, F. Abdullah, An open-access microfluidic model for lung-specific functional studies at an air-liquid interface, Biomed Microdevices, 11 (2009) 1081-1089.

[16] D. Huh, B.D. Matthews, A. Mammoto, M. Montoya-Zavala, H.Y. Hsin, D.E. Ingber, Reconstituting organ-level lung functions on a chip, Science, 328 (2010) 16621668

[17] D. Huh, D.C. Leslie, B.D. Matthews, J.P. Fraser, S. Jurek, G.A. Hamilton, K.S. Thorneloe, M.A. McAlexander, D.E. Ingber, A human disease model of drug toxicityinduced pulmonary edema in a lung-on-a-chip microdevice, Sci Transl Med, 4 (2012) 159 ra147.

[18] Y. Imura, Y. Asano, K. Sato, E. Yoshimura, A microfluidic system to evaluate intestinal absorption, Anal Sci, 25 (2009) 1403-1407.

[19] Y. Imura, K. Sato, E. Yoshimura, Micro total bioassay system for ingested substances: assessment of intestinal absorption, hepatic metabolism, and bioactivity, 
Anal Chem, 82 (2010) 9983-9988.

[20] Y. Imura, E. Yoshimura, K. Sato, Microcirculation system with a dialysis part for bioassays evaluating anticancer activity and retention, Anal Chem, 85 (2013) 1683-1688. [21] H.J. Kim, D. Huh, G. Hamilton, D.E. Ingber, Human gut-on-a-chip inhabited by microbial flora that experiences intestinal peristalsis-like motions and flow, Lab Chip, 12 (2012) 2165-2174.

[22] D.K. Wood, A. Soriano, L. Mahadevan, J.M. Higgins, S.N. Bhatia, A biophysical indicator of vaso-occlusive risk in sickle cell disease, Sci Transl Med, 4 (2012) 123 ra126.

[23] J.Q. Yu, X.F. Liu, L.K. Chin, A.Q. Liu, K.Q. Luo, Study of endothelial cell apoptosis using fluorescence resonance energy transfer (FRET) biosensor cell line with hemodynamic microfluidic chip system, Lab Chip, 13 (2013) 2693-2700.

[24] H. Hashizume, P. Baluk, S. Morikawa, J.W. McLean, G. Thurston, S. Roberge, R.K. Jain, D.M. McDonald, Openings between defective endothelial cells explain tumor vessel leakiness, Am J Pathol, 156 (2000) 1363-1380.

[25] L. Chau, M. Doran, J. Cooper-White, A novel multishear microdevice for studying cell mechanics, Lab Chip, 9 (2009) 1897-1902.

[26] K.M. Chrobak, D.R. Potter, J. Tien, Formation of perfused, functional microvascular tubes in vitro, Microvasc Res, 71 (2006) 185-196.

[27] M.B. Esch, D.J. Post, M.L. Shuler, T. Stokol, Characterization of in vitro endothelial linings grown within microfluidic channels, Tissue Eng Part A, 17 (2011) 2965-2971.

[28] C. Fidkowski, M.R. Kaazempur-Mofrad, J. Borenstein, J.P. Vacanti, R. Langer, Y. Wang, Endothelialized microvasculature based on a biodegradable elastomer, Tissue 
Eng, 11 (2005) 302-309.

[29] J. Shao, L. Wu, J. Wu, Y. Zheng, H. Zhao, Q. Jin, J. Zhao, Integrated microfluidic chip for endothelial cells culture and analysis exposed to a pulsatile and oscillatory shear stress, Lab Chip, 9 (2009) 3118-3125.

[30] A.D. van der Meer, A.A. Poot, J. Feijen, I. Vermes, Analyzing shear stress-induced alignment of actin filaments in endothelial cells with a microfluidic assay, Biomicrofluidics, 4 (2010) 11103.

[31] J.H. Yeon, H.R. Ryu, M. Chung, Q.P. Hu, N.L. Jeon, In vitro formation and characterization of a perfusable three-dimensional tubular capillary network in microfluidic devices, Lab Chip, 12 (2012) 2815-2822.

[32] Y. Tanaka, Y. Kikukawa, K. Sato, Y. Sugii, T. Kitamori, Culture and leukocyte adhesion assay of human arterial endothelial cells in a glass microchip, Anal Sci, 23 (2007) 261-266.

[33] R. Estrada, G.A. Giridharan, M.D. Nguyen, T.J. Roussel, M. Shakeri, V. Parichehreh, S.D. Prabhu, P. Sethu, Endothelial cell culture model for replication of physiological profiles of pressure, flow, stretch, and shear stress in vitro, Anal Chem, 83 (2011) 3170-3177.

[34] A.P. Golden, J. Tien, Fabrication of microfluidic hydrogels using molded gelatin as a sacrificial element, Lab Chip, 7 (2007) 720-725.

[35] J.W. Song, S.P. Cavnar, A.C. Walker, K.E. Luker, M. Gupta, Y.C. Tung, G.D. Luker, S. Takayama, Microfluidic endothelium for studying the intravascular adhesion of metastatic breast cancer cells, PLoS One, 4 (2009) e5756.

[36] J.W. Song, W. Gu, N. Futai, K.A. Warner, J.E. Nor, S. Takayama, Computercontrolled microcirculatory support system for endothelial cell culture and shearing, 
Anal Chem, 77 (2005) 3993-3999.

[37] G.M. Price, K.H. Wong, J.G. Truslow, A.D. Leung, C. Acharya, J. Tien, Effect of mechanical factors on the function of engineered human blood microvessels in microfluidic collagen gels, Biomaterials, 31 (2010) 6182-6189.

[38] S. Lindstrom, K. Mori, T. Ohashi, H. Andersson-Svahn, A microwell array device with integrated microfluidic components for enhanced single-cell analysis, Electrophoresis, 30 (2009) 4166-4171.

[39] P.A. Vogel, S.T. Halpin, R.S. Martin, D.M. Spence, Microfluidic transendothelial electrical resistance measurement device that enables blood flow and postgrowth experiments, Anal Chem, 83 (2011) 4296-4301.

[40] E.W. Young, M.W. Watson, S. Srigunapalan, A.R. Wheeler, C.A. Simmons, Technique for real-time measurements of endothelial permeability in a microfluidic membrane chip using laser-induced fluorescence detection, Anal Chem, 82 (2010) 808816.

[41] D.D. Nalayanda, C.M. Puleo, W.B. Fulton, T.H. Wang, F. Abdullah, Characterization of pulmonary cell growth parameters in a continuous perfusion microfluidic environment, Exp Lung Res, 33 (2007) 321-335.

[42] D.D. Nalayanda, Q. Wang, W.B. Fulton, T.H. Wang, F. Abdullah, Engineering an artificial alveolar-capillary membrane: a novel continuously perfused model within microchannels, J Pediatr Surg, 45 (2010) 45-51.

[43] M. Shin, K. Matsuda, O. Ishii, H. Terai, M. Kaazempur-Mofrad, J. Borenstein, M. Detmar, J.P. Vacanti, Endothelialized networks with a vascular geometry in microfabricated poly(dimethyl siloxane), Biomed Microdevices, 6 (2004) 269-278.

[44] N. Sasaki, M. Shinjo, S. Hirakawa, M. Nishinaka, Y. Tanaka, K. Mawatari, T. 
Kitamori, K. Sato, A palmtop-sized microfluidic cell culture system driven by a miniaturized infusion pump, Electrophoresis, 33 (2012) 1729-1735.

[45] L.K. Chin, J.Q. Yu, Y. Fu, T. Yu, A.Q. Liu, K.Q. Luo, Production of reactive oxygen species in endothelial cells under different pulsatile shear stresses and glucose concentrations, Lab Chip, 11 (2011) 1856-1863.

[46] N.J. Douville, Y.C. Tung, R. Li, J.D. Wang, M.E. El-Sayed, S. Takayama, Fabrication of two-layered channel system with embedded electrodes to measure resistance across epithelial and endothelial barriers, Anal Chem, 82 (2010) 2505-2511.

[47] B. Prabhakarpandian, M.C. Shen, J.B. Nichols, I.R. Mills, M. SidorykWegrzynowicz, M. Aschner, K. Pant, SyM-BBB: a microfluidic blood brain barrier model, Lab Chip, 13 (2013) 1093-1101.

[48] C.P. Ng, S.H. Pun, A perfusable 3D cell-matrix tissue culture chamber for in situ evaluation of nanoparticle vehicle penetration and transport, Biotechnol Bioeng, 99 (2008) 1490-1501.

[49] S. Chung, R. Sudo, P.J. Mack, C.R. Wan, V. Vickerman, R.D. Kamm, Cell migration into scaffolds under co-culture conditions in a microfluidic platform, Lab Chip, 9 (2009) 269-275.

[50] S. Chung, R. Sudo, I.K. Zervantonakis, T. Rimchala, R.D. Kamm, Surfacetreatment-induced three-dimensional capillary morphogenesis in a microfluidic platform, Adv Mater, 21 (2009) 4863-4867.

[51] G.S. Jeong, S. Han, Y. Shin, G.H. Kwon, R.D. Kamm, S.H. Lee, S. Chung, Sprouting angiogenesis under a chemical gradient regulated by interactions with an endothelial monolayer in a microfluidic platform, Anal Chem, 83 (2011) 8454-8459. [52] J.W. Song, L.L. Munn, Fluid forces control endothelial sprouting, Proc Natl Acad 
Sci U S A, 108 (2011) 15342-15347.

[53] E. Tkachenko, E. Gutierrez, M.H. Ginsberg, A. Groisman, An easy to assemble microfluidic perfusion device with a magnetic clamp, Lab Chip, 9 (2009) 1085-1095. [54] P.L. Voyvodic, D. Min, A.B. Baker, A multichannel dampened flow system for studies on shear stress-mediated mechanotransduction, Lab Chip, 12 (2012) 3322-3330. [55] T. Herricks, K.B. Seydel, G. Turner, M. Molyneux, R. Heyderman, T. Taylor, P.K. Rathod, A microfluidic system to study cytoadhesion of Plasmodium falciparum infected erythrocytes to primary brain microvascularendothelial cells, Lab Chip, 11 (2011) 2994-3000.

[56] A.L. Bowen, R.S. Martin, Integration of on-chip peristaltic pumps and injection valves with microchip electrophoresis and electrochemical detection, Electrophoresis, 31 (2010) 2534-2540.

[57] R. Gomez-Sjoberg, A.A. Leyrat, D.M. Pirone, C.S. Chen, S.R. Quake, Versatile, fully automated, microfluidic cell culture system, Anal Chem, 79 (2007) 8557-8563.

[58] N. Futai, W. Gu, J.W. Song, S. Takayama, Handheld recirculation system and customized media for microfluidic cell culture, Lab Chip, 6 (2006) 149-154.

[59] J.K. Tsou, R.M. Gower, H.J. Ting, U.Y. Schaff, M.F. Insana, A.G. Passerini, S.I. Simon, Spatial regulation of inflammation by human aortic endothelial cells in a linear gradient of shear stress, Microcirculation, 15 (2008) 311-323.

[60] I.K. Zervantonakis, S.K. Hughes-Alford, J.L. Charest, J.S. Condeelis, F.B. Gertler, R.D. Kamm, Three-dimensional microfluidic model for tumor cell intravasation and endothelial barrier function, Proc Natl Acad Sci U S A, 109 (2012) 13515-13520.

[61] Q. Zhang, T. Liu, J. Qin, A microfluidic-based device for study of transendothelial invasion of tumor aggregates in realtime, Lab Chip, 12 (2012) 2837-2842. 
[62] N. Korin, M. Kanapathipillai, B.D. Matthews, M. Crescente, A. Brill, T. Mammoto, K. Ghosh, S. Jurek, S.A. Bencherif, D. Bhatta, A.U. Coskun, C.L. Feldman, D.D. Wagner, D.E. Ingber, Shear-activated nanotherapeutics for drug targeting to obstructed blood vessels, Science, 337 (2012) 738-742.

[63] L.M. Griep, F. Wolbers, B. de Wagenaar, P.M. ter Braak, B.B. Weksler, I.A. Romero, P.O. Couraud, I. Vermes, A.D. van der Meer, A. van den Berg, BBB on chip: microfluidic platform to mechanically and biochemically modulate blood-brain barrier function, Biomed Microdevices, 15 (2013) 145-150.

[64] R. Booth, H. Kim, Characterization of a microfluidic in vitro model of the bloodbrain barrier (muBBB), Lab Chip, 12 (2012) 1784-1792. 
a
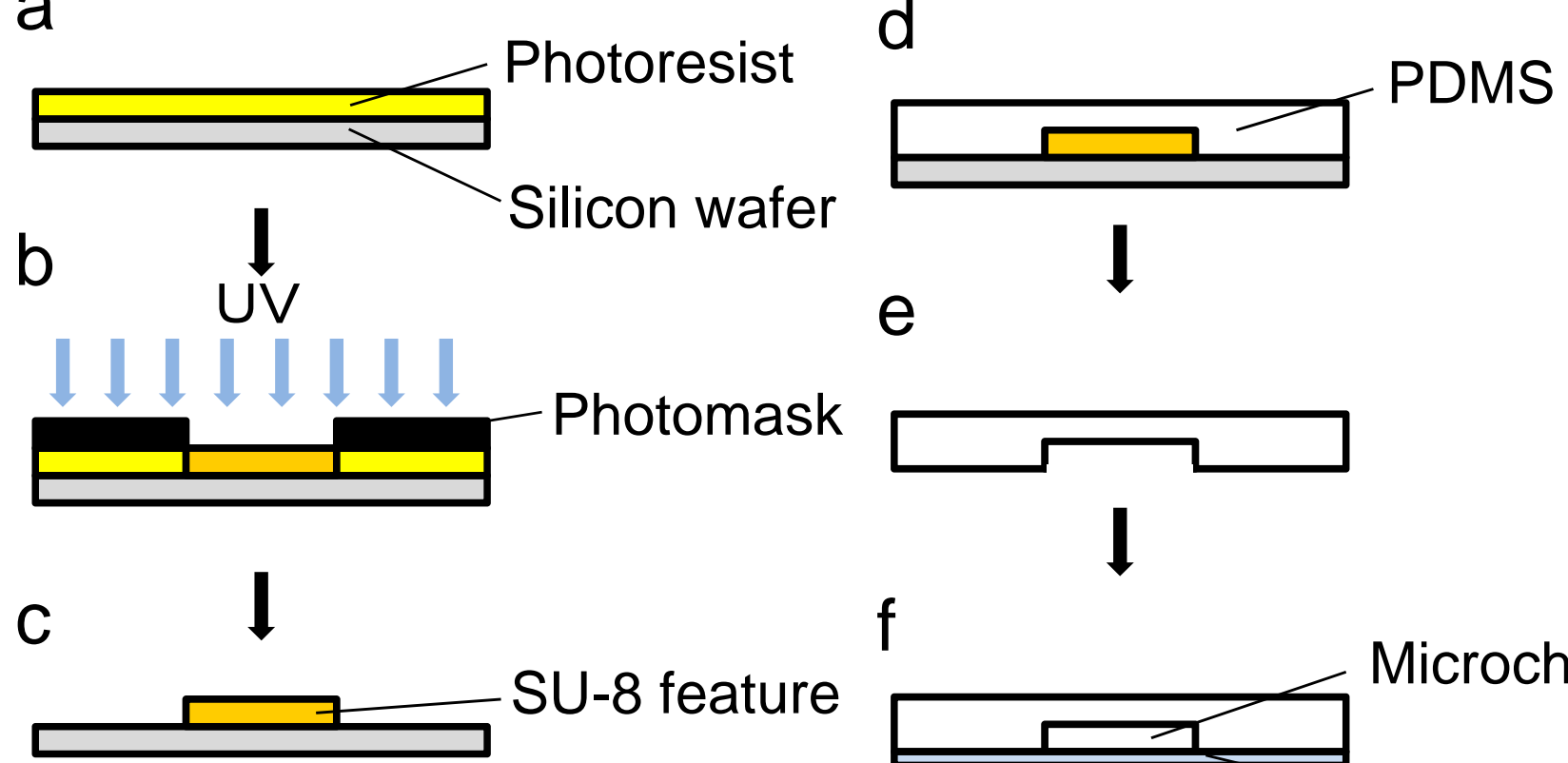

f

Microchannel

Glass slide

Figure 1

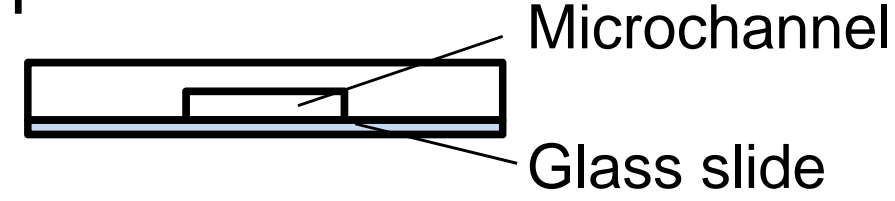



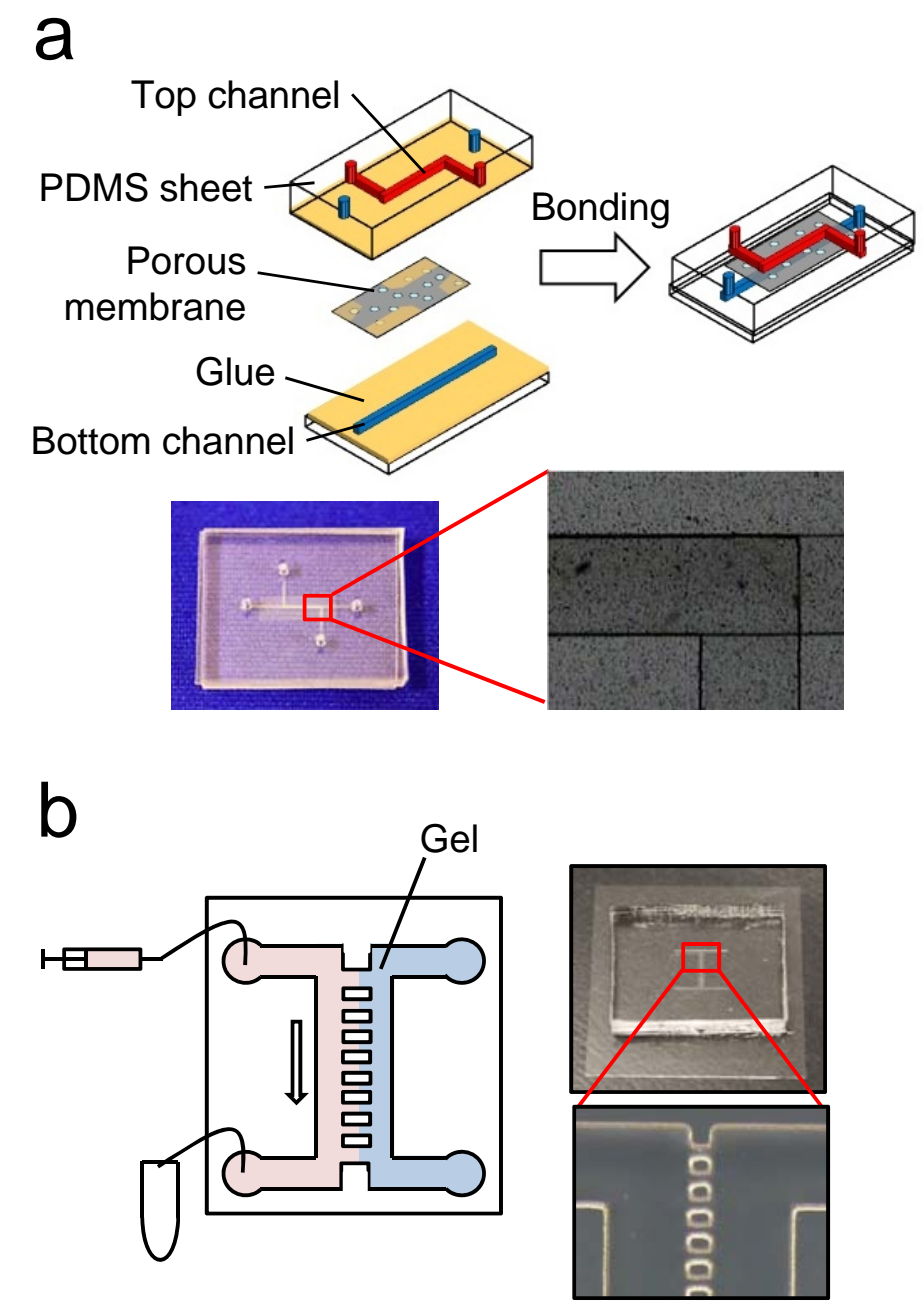

Figure 2 
a

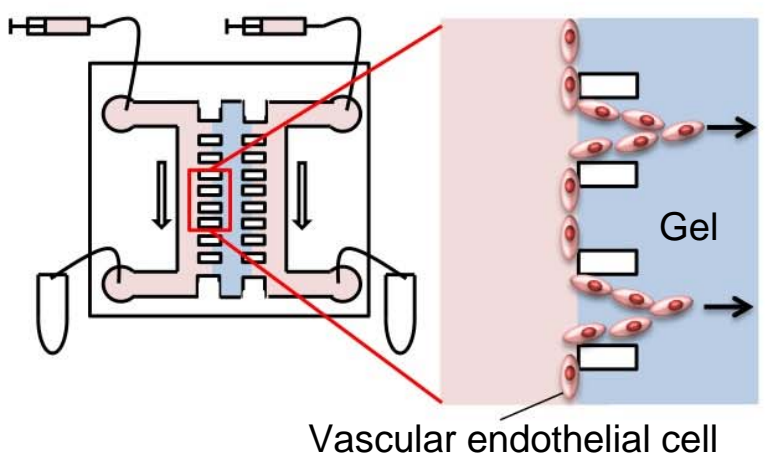

b

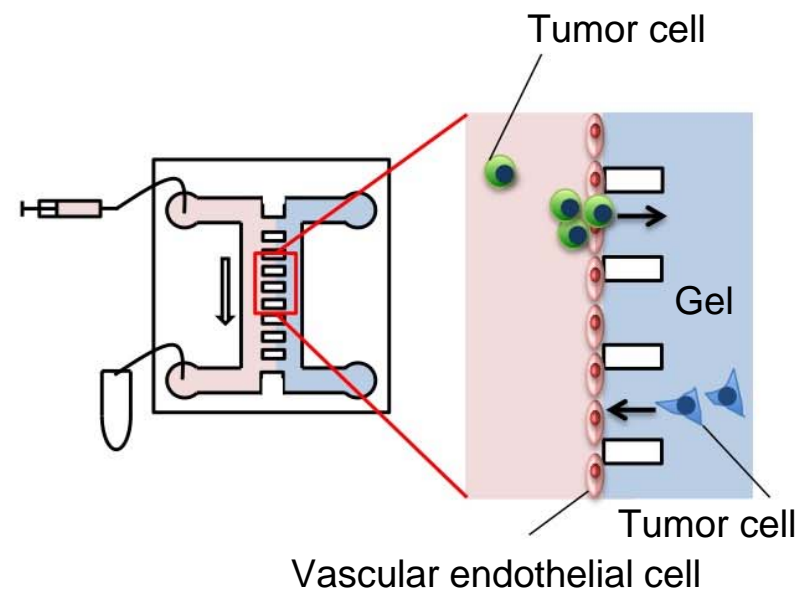

C

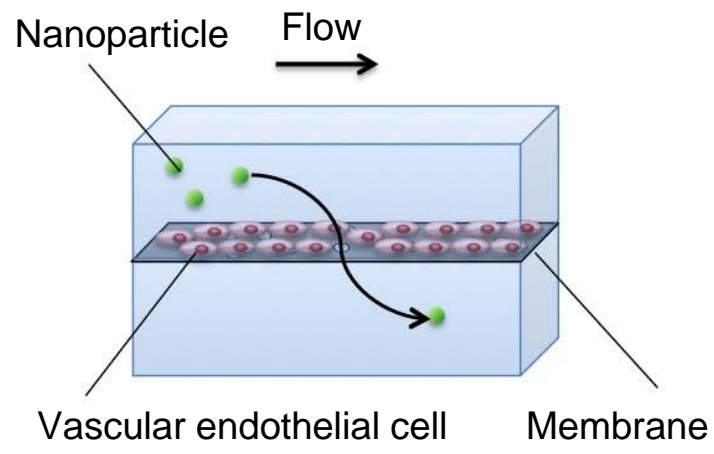

d

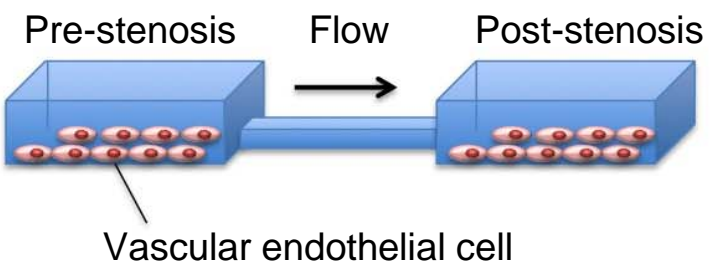

Figure 3 


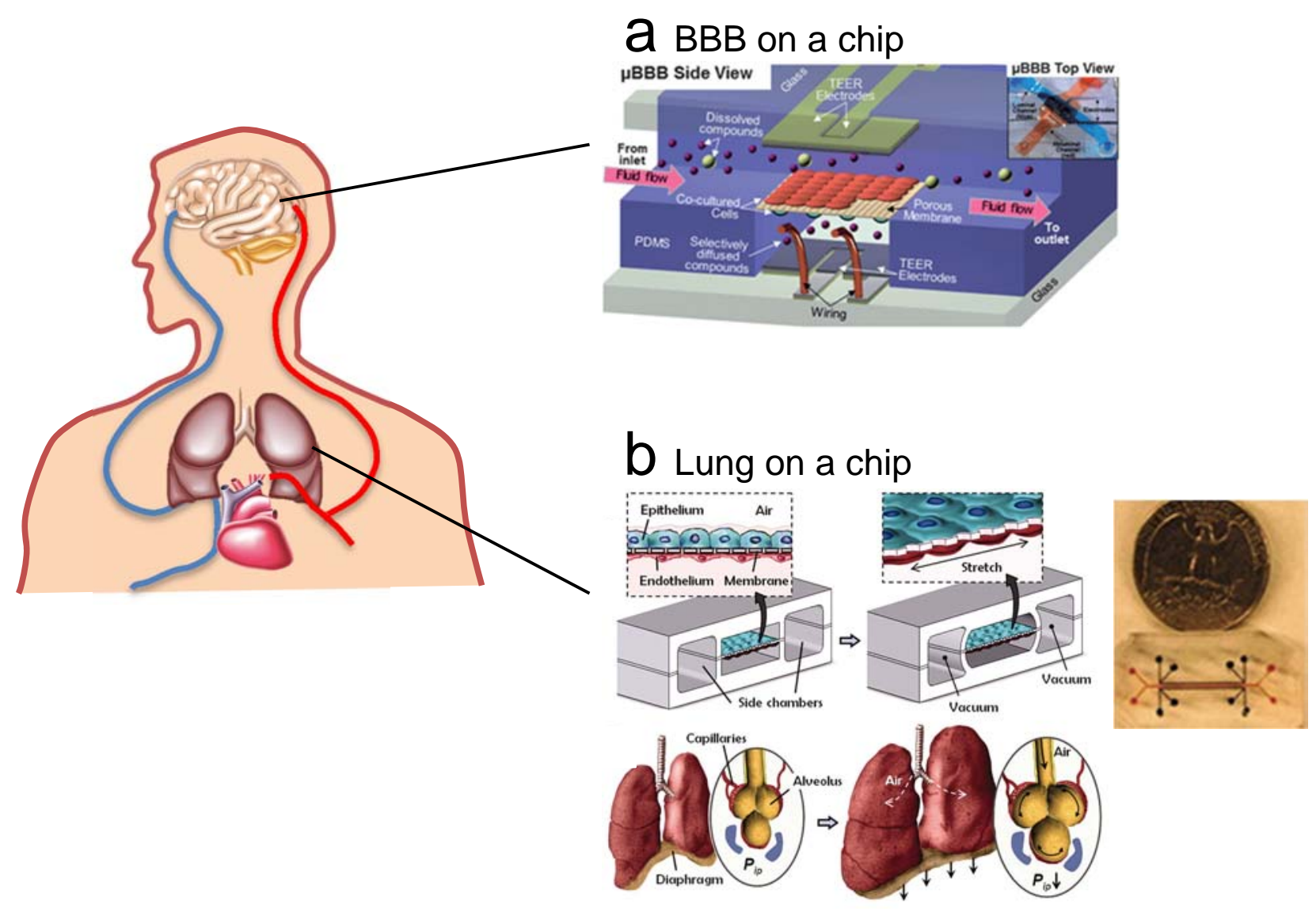


